\title{
GROWTH PERFORMANCE AND CARCASS CHARACTERISTICS OF BARKI SHEEP AS AFFECTED BY FLAVOMYCIN AND DRINKING SALINE WATER
}

\author{
M.F. Shehata and A.H. Hammam
}

Department of Animal and Poultry Breeding, Desert Research Center, Matareya, Cairo, Egypt

\section{SUMMARY}

Forty Barki lambs weighed $26.04 \pm 0.81 \mathrm{~kg}$ aged six months old were used to investigate the effect of flavomycin as growth promoter and drinking saline water on their growth performance and carcass charateristics. Animals were divided into four equal groups of similar average body weight and assigned to factorial experiment (2x2). Basal diet was adopted to cover requriments of $100 \mathrm{gm}$ daily gain with or without $20 \mathrm{mg} / \mathrm{h} / \mathrm{d}$ flavomycin in addition to either tap water, TW (505ppm TDS) or saline water, SW (12494 ppm TDS) for drinking. This experiment lasted for 180 days as a feeding trial followed by a slaughter experiment on three animal from each group to study of carcass traits.

Flavomycin increased efficiency of feed conversion, but had no effect on body weight changes. Drinking SW decreased daily body weight gain, total body weight gain, nutrients intake and feed efficiency. Adding flavomycin reduced these negative effects of drinking $S W$ on growth performance.

Drinking tap water improved feed conversion than that with salty water (11.13 vs $9.45 \%$ ). Dressing percentage calculated relative to either slaughter weight or empty body weight was higher with drinking tap water than salty water (43.65, 53.01 vs $39.05,50.05 \%$, respectively). Drinking $S W$ resulted in decreasing empty body weight and hot carcass weight by 64 and $2.40 \mathrm{~kg}$, respectively, in addition to a significant decrease in dressing percentage by $2.95 \%$ compared to the control group. Adding flavomycin to the animals that drank $S w$ moderated that decrease to become $1.62 \mathrm{~kg}, 1.90 \mathrm{~kg}$ and $2.38 \%$ respectively. Drinking $S W$ increased the lean percentage in the best rib cut on account of the fat percentage. Flavomycin overcame the negative effects of $S W$ on the spleen weight. It was concluded that adding flavomycin to growing lambs could reduce some negative effects of drinking $S W$ on growth performance and carcass traits.

Keywords:Lambs, flavomycin, saline water, performance, carcass traits

\section{INTRODUCTION}

The vital need for the animal protein in Egypt has created the interest towards increasing the growth performance by different means, including utilization of the growth promoters. Feeding flavomycin increased feed efficiency and rate of gain of sheep (Murray et al, 1992; and Paitil et al., 1996). Flavomycin is the trade name for the antibiotic performance promoter "Flavophosphplipol" and predominantly in vitro against gram-positive pathogenic bacteria. Flavomycin proved to be less effective 
against gram-negative pathogenic micro-organisms. The antibiotic mode of action of flavomycin is based on an inhibition of the biosynthesis of the bacterial cell wall; this follows from the fact that cell wall precursors accumulate in the presence of Flavomycin.

In coastal desert areas, the drinking water available for animals is mainly well water. The salinity of well water along these areas was found to vary from 360 to $30082 \mathrm{ppm}$ total dissolved salts (Aggour, 1990). Pierce (1966) noted that sheep that drank water containing up to $1.5 \%$ sodium chloride without ill effects, yet the $2 \%$ level was detrimental. El-Sherif and El-Hassanein (1996) reported that long-term administration of saline water decreased growth rate and final body weight of Barki ram lambs. However, drinking saline water within the tolerance level did not adversely affect live weight gain of sheep (Singh and Taneja, 1979). Further more, sheep are exposed to harsh climatic conditions and feed shortage in Semi - arid areas, which would affect badly their growth performance (Aboul-Nage and Aboul-Ela, 1986 and El-Sherif, 1991). Ahmed (1996) reported that the average litter size was 1.02 and lamb survival rate up to weaning was 0.77 . All these characteristics lead to low biological feed efficiency for meat production, which ranges between 22.7 and $27.0 \mathrm{~kg} \mathrm{DM} / \mathrm{kg} \mathrm{BW}$ (Abd-El-Aziz, 1983).

In addition, significant improvement in feed conversion efficiency was found during the growth period of lambs (El-Basiony, 1994 and El-Sherif et al, 2001).

Sheep are among the domestic animals Kept for meat and milk production. From this point of view, nowadays the new trends are to increase the productivity and metabolic efficiency. The present study was undertaken to investigate the effect of the fiavomycin as growth promtor with fresh tap or saline water on performance and carcass traits of Barki Sheep.

\section{MATERIALS AND METHODS}

This study was carried out in Maryout Research Station, belongs to the Desrert Research Center, Egypt.

\section{A-Animals and treatments :}

Forty Barki male lambs averaged $26.04 \pm 0.80 \mathrm{Kg}$ live body weight aged were used in this study. Lambs were divided into four equal groups (10 animals each) of similar average body weight and allocated to four treatments i.e. (1) Basal diet and fresh tap water for drinking without flavomycin, (2) Basal diet and fresh tap water for drinking with $20 \mathrm{mg} / \mathrm{h} / \mathrm{d}$ flavomycin, (3) Basal diet and saline water for drinking without flavomycin and (4) Basal diet and saline water for drinking with $20 \mathrm{mg} / \mathrm{h} / \mathrm{d}$ flavomycin. Hammam, A.H (2001) found that the best level of flavomycin from the economic point of view was $20 \mathrm{mg} / \mathrm{h} / \mathrm{d}$, since the gave the highest amount of live body gain and the least cost for each kilogram gain.

Saline water was obtained by diluting sea water with fresh tap water in ratio of 1:2, respectively. Fresh tap water had $505 \mathrm{ppm}$ total dissolved salts (TDS), while saline water had $12494 \mathrm{ppm}$ TDS (Table 1). The level of salinity was below the salt tolerance level of sheep reported by Pierce, (1966). The TDS was measured using electrical conductivity apparatus. Flavomycin (a growth promoter) was orally given to animals as a suspension using a drenching gun. Drinking water was available $a d$ 
libitum for the animals two times daily. Animals were housed in shaded pens $(4 \times 6$ meters) roofed with asbestos sheets at the height of 5 meters.

Table 1. Chemical composition of feed ingredients in ration of sheep

\begin{tabular}{llllllll}
\hline Item & DM & OM & CP & CF & EE & Ash & NFE \\
\cline { 3 - 7 } & & \multicolumn{5}{c}{ DM basis, \% } \\
\hline CFM $^{*}$ & 84.85 & 92.40 & 13.91 & 12.11 & 2.99 & 7.60 & 63.39 \\
Barley grains & 87.65 & 95.55 & 9.11 & 6.31 & 1.92 & 4.45 & 78.21 \\
Berseem hay & 90.65 & 85.57 & 12.31 & 28.31 & 2.19 & 14.43 & 42.76 \\
\hline
\end{tabular}

* Concentrate feed mixture.

B-Feeding experiment :

At the beginning of the feeding experiment, all animals were drenched to control internal parasites. The feeding experiment extended for 180 .

Levels of feeding were calculated according to Kearl, (1982) to cover nutritional requirements of $100 \mathrm{~g}$ gain/day.. All groups were given the concentrates and berseem hay at the ratio of 1:1 of TDN (basal diet). The concentrate portion consisted of barley grains and concentrate feed mixture (CFM) at ratio of 1:3, respectively. The CFM consisted of cotton seed cake $50 \%$, wheat bran $18 \%$, yellow maize $15 \%$, rice polish $11 \%$, molasses $3 \%$, limestone $2 \%$ and common salt $1 \%$. Actual feed intake was measured and weekly samples of feedstuffs were collected and analyzed according to A.O.A.C. (1990). Chemical composition of different feedstuffs is presented in Table (1). Live body weight changes $(\mathrm{Kg})$ of each animal was recorded biweekly during the feeding experiment.

The level of nutrient requirements was adjusted according to changes of live body weight. Daily feed intake as a dry matter (DM) and total digestible nutrients (TDN) was calculated and expressed either as absolute values $(\mathrm{g} / \mathrm{h} / \mathrm{d})$. Efficiency of feed conversion (EFC) was calculated according to the following equations:

$\mathrm{EFC} 1=($ average daily gain $\mathrm{X} 100) /$ average daily dry matter intake

$\mathrm{EFC} 2=($ average daily gain X 100) / average daily digestible nutrients intake

\section{C-Slaughter experiment :}

At the end of the feeding period, three lambs of each group were chosen representing the average live body weight of the group and slaughtered after a fasting period of $18 \mathrm{hr}$ to evalute carcass traits. After slaughter and skinning, weights of all organs and offals were recorded. The digestive tract was weighed full and empty to obtain weight of its contents. Empty body weight (EBW) was calculated by subtracting weight of gut fill from slaughter weight. Hot carcass weight was determined immediately after devisceration.

Eash carcass was cut into seven joints, i.e. legs, loin, racks, flank, neck and tail according to the Egyptian wholesale mutton cuts (Hamada, 1976).

The right 9-10-11 rib cuts were physically separated into their components, i.e. lean, fat and bone and expressed as percentage of the weight of the whole cut.

\section{D- Organoleptic test:}

A taste panel was performed to assess the organoleptic traits of the meat in terms of aroma, flavour, tenderness, juiciness and palatability. Samples from loin cut of each lamb were cooked (boiled for 45 minutus) and served to 10 panelists in Maryout Research Station. Each trait was scored on a scale from 1 to 5 representing the grades of very poor, poor, fair, good and very good, respectively. 


\section{E-Statistical Analysis:}

Data of the effect of saline water and flavomycin administration were analyzed using SAS software (SAS, 1985). Arcsine values were used for statistical analysis of the paramerters caculated in percentages.

\section{RESULTS AND DISCUSSION}

\section{Animals performance:}

Least-squares means of initial and final body wt, daily gain, feed intake and feed conversion efficiency of different animal groups are presented in Table (3). Body weight changes of the groups that drank fresh water were 19.83 and $18.45 \mathrm{Kg} / \mathrm{head}$ for no flavomycin and flavomycin given groups, respectively. These values were higher $(\mathrm{P}<0.05)$ than corresponding groups that drank saline water $(16.28$ and 16.70 $\mathrm{Kg} /$ head, respectively).

Table 2. Total dissolved solids and some elements (ppm) in different types of water

\begin{tabular}{lccccc}
\hline Type of water & TDS & Na & K & \multicolumn{1}{l}{ Mg } & \multicolumn{1}{c}{ Cl } \\
\hline Tap water & 505 & 180 & 11 & 19 & 220 \\
Sea water & 36480 & 12000 & 550 & 1312 & 13165 \\
Diluted sea water & 12494 & 4122 & 191.9 & 453 & 4532 \\
\hline
\end{tabular}

Table 3: Least square means $\pm \mathrm{SE}$ of initial body wt, final body wt, daily gain, dry matter intake and feed efficiency for lambs in experimental groups

\begin{tabular}{|c|c|c|c|c|c|c|c|}
\hline \multirow[t]{2}{*}{ Item } & \multirow{2}{*}{$\begin{array}{c}\text { FL } \\
\text { Level }\end{array}$} & \multicolumn{2}{|c|}{ Type of water } & \multirow{2}{*}{$\begin{array}{c}\text { Mean } \\
\text { FL }\end{array}$} & \multicolumn{3}{|c|}{$\pm \mathbf{S E}$} \\
\hline & & TW & SW & & $\mathbf{W}$ & FL & $\mathbf{W} \square \mathbf{F L}$ \\
\hline \multirow[t]{3}{*}{ Initial body wt (kg) } & FL 00 & 27.11 & 25.85 & 26.48 & 1.17 & 1.17 & 1.65 \\
\hline & FL 20 & 25.70 & 25.60 & 25.65 & ns & ns & ns \\
\hline & Mean W & 26.41 & 25.73 & & & & \\
\hline \multirow[t]{3}{*}{ Finial body wt (kg) } & FL 00 & 46.94 & 42.13 & 44.66 & 1.73 & 1.73 & 2.44 \\
\hline & FL 20 & 44.15 & 42.30 & 43.23 & ns & ns & ns \\
\hline & Mean W & 45.55 & 42.22 & & & & \\
\hline \multirow[t]{3}{*}{ Total body gain $(\mathrm{kg})$} & FL 00 & 19.83 & 18.28 & 18.06 & 0.99 & 0.94 & 1.41 \\
\hline & FL 20 & 18.45 & 16.70 & 17.58 & $*$ & ns & ns \\
\hline & Mean W & 19.14 & 16.49 & & & & \\
\hline \multirow[t]{3}{*}{ Daily gain $(\mathrm{g} / \mathrm{h} / \mathrm{d})$} & FL 00 & 116.45 & 92.51 & 104.48 & 3.90 & 3.90 & 5.41 \\
\hline & FL 20 & 112.31 & 100.15 & 106.23 & $* *$ & ns & ns \\
\hline & Mean W & 114.38 & 96.33 & & & & \\
\hline \multirow[t]{3}{*}{ DMI $g / h / d$} & FL 00 & 1054.2 & 969.8 & 1012.0 & 3.59 & 3.58 & 5.07 \\
\hline & FL 20 & 1017.2 & 981.9 & 999.6 & ns & ns & $* *$ \\
\hline & Mean W & 1035.7 & 975.8 & & & & \\
\hline \multirow[t]{3}{*}{$\operatorname{TDN}(\mathrm{g} / \mathrm{h} / \mathrm{d})$} & FL 00 & 699.03 & 643.15 & 671.09 & 2.38 & 2.38 & 3.37 \\
\hline & FL 20 & 675.46 & 651.00 & 663.23 & ns & ns & $* *$ \\
\hline & Mean W & 687.25 & 647.08 & & & & \\
\hline \multirow[t]{3}{*}{ DM conversion } & FL 00 & 11.13 & 9.45 & 10.29 & 0.39 & 0.39 & 0.56 \\
\hline & FL 20 & 11.36 & 10.31 & 10.83 & $* *$ & ns & $\mathrm{ns}$ \\
\hline & Mean W & 11.24 & 9.38 & & & & \\
\hline \multirow[t]{3}{*}{ TDN conversion } & FL 00 & 16.78 & 14.26 & 15.52 & 0.59 & 0.59 & 0.84 \\
\hline & FL 20 & 17.11 & 15.55 & 16.33 & $* *$ & ns & ns \\
\hline & Mean W & 16.94 & 16.78 & & & & \\
\hline
\end{tabular}

FW: Fresh water; SW: Saline water; FW x FL: Fresh water + Flavomycin; SW x FL : Saline water +

Flavomycin; $\quad *$ : $\mathrm{P}<0.05$; **: $\mathrm{P}<0.01$; ns: not significant $\mathrm{P}<0.05$ 
Drinking SW resulted in significant decrease in total body weight gain and average daily gain. El-Sherif and El-Hassanein (1996) obtained same results with growing Barki ram lambs after prolonged administration of SW. On the contrary, Shawkat et al. (1988) reported an increase in live body weight of sheep and camels by drinking SW as a result of increased water intake.

Dry matter or TDN intakes (either absolute weight or relative to metabolic body size) of animal groups were not significantly affected by either flavomycin or salinity of water. In the present work, there was a minor decrease in different nutrients intake by SW, but more obvious in the absolute values (Table 3 ).

These results of intake and body gain resulted in a significant $(\mathrm{P}<0.01)$ decrease of feed efficiency (EFC) group drinking SW. However, administration of FL to the animals drinking SW reduced these negative effects on feed intake and feed conversion . Similarly, El-Basiony (1994) reported that flavomycin improved feed conversion ratios during the growth period of Ossimi sheep. This indicated the role of FL as antistressor as mentioned by Davey (1980). In this context, Ismail (2003) stated that drinking saline water was found to be stressor on nutrient utlilization as it decreased most of the digestibility coefficients.

\section{Carcass data:}

Least-squares means of slaughter weight, empty body weight, hot carcass weight, and dressing percentage of lambs for different experimental groups are shown in Table (4). There were no significant effects due to either SW or FL on empty body weight (EBW) and hot carcass weight (HCW). Dressing percentage (DP\%) decreased $(\mathrm{P}<0.05)$ due to drinking SW. Fahmy (1998) reported that DP\% of sheep did not change by saline load through feeding silage of salt bushes a basal diet. Mohamed (1997) reported significant decrease in HCW and DP\% of both California and NewZealand White rabbits by drinking SW. Ahmed (1996) suggested that the decrease in weights of carcass and its components by saline load could be due to depression in final body weight and body solids content.

In cattle, Spires et al. (1990) noted that DP\% was greater when using FL at a rate of $30 \mathrm{mg} / \mathrm{head} /$ day as compared to rate of $45 \mathrm{mg} / \mathrm{h} /$ day (68 vs. $66.8 \%$ ). However, Morris et al. (1990) reported that antibiotic growth promoters had no effect on D\% and carcass composition. Although insignificant, there was a substantial decrease in EBW and HCW by drinking SW effects equated to $2.64 \mathrm{~kg}$ and $2.40 \mathrm{~kg}$, respectively, in addition to a significant decrease in dressing percentage by $2.95 \%$. Adding FL to the animals diet that drank SW moderated that decrease to become only $1.62 \mathrm{~kg}, 1.9$ and $2.38 \%$ in EBW, $\mathrm{HCW}, \mathrm{D} \%$, respectively.

\section{Organs and offals :}

Table (5) illustrates the effect of SW and FL on the different organs and ofals expressed as a percentage of EBW. Drinking SW decreased significantly spleen and heart percentages. Spleen was the only organ that was affected by flavomycin addition $(\mathrm{P}<0.01)$ and the interaction between salinity of water and flavomycin $(\mathrm{P}<0.01)$. It seems that the effect of salt water caused shrinkage in spleen that made it less in weight. Flavomycin diluted the negative effect of SW on the spleen size hence there was significant interaction of SW X FL on this organ. 
The relation between spleen and drinking salt water is not clear. Ayyat et al. (1991) reported that drinking saline water $(3000,4500$ and $6000 \mathrm{ppm}$ salt) caused a significant decrease in kidney fat in rabbits.

Table 4. Least square means $\pm \mathrm{SE}$ of initial body wt, slaughter wt, empty body wt, hot carcass wt, chilled carcass wt and dressing percentage for lambs in experimental groups

\begin{tabular}{|c|c|c|c|c|c|c|c|}
\hline \multirow[t]{2}{*}{ Item } & \multirow{2}{*}{$\begin{array}{c}\text { FL } \\
\text { Level }\end{array}$} & \multicolumn{2}{|c|}{ Type of water } & \multirow{2}{*}{$\begin{array}{c}\text { Mean } \\
\text { FL }\end{array}$} & \multicolumn{3}{|c|}{$\pm \mathbf{S E}$} \\
\hline & & TW & SW & & $\mathbf{W}$ & FL & $\mathbf{W} \square \mathbf{F L}$ \\
\hline \multirow[t]{3}{*}{ Initial body wt (kg) } & FL 00 & 27.11 & 25.85 & 26.48 & 1.17 & 1.17 & 1.65 \\
\hline & FL 20 & 25.70 & 25.60 & 25.65 & ns & ns & ns \\
\hline & Mean W & 26.41 & 25.73 & & & & \\
\hline \multirow[t]{3}{*}{ Slaughter wt (kg) } & FL 00 & 46.94 & 42.13 & 44.66 & 1.73 & 1.73 & 2.44 \\
\hline & FL 20 & 44.15 & 42.30 & 43.23 & ns & ns & ns \\
\hline & Mean W & 45.55 & 42.22 & & & & \\
\hline \multirow[t]{3}{*}{ Empty body wt (kg) } & FL 00 & 37.71 & 35.08 & 36.40 & 2.81 & 2.81 & 3.97 \\
\hline & FL 20 & 35.83 & 36.08 & 35.96 & ns & ns & ns \\
\hline & Mean W & 36.77 & 35.58 & & & & \\
\hline \multirow[t]{3}{*}{ Hot carcass wt $(\mathrm{kg})$} & FL 00 & 20.07 & 17.67 & 18.87 & 1.58 & 1.58 & 2.23 \\
\hline & FL 20 & 18.97 & 18.17 & 18.57 & ns & ns & $\mathrm{ns}$ \\
\hline & Mean W & 19.52 & 17.92 & & & & \\
\hline \multirow[t]{3}{*}{ Chilled carcass wt (kg) } & FL 00 & 19.37 & 16.43 & 17.90 & 1.48 & 1.48 & 2.09 \\
\hline & FL 20 & 18.17 & 16.83 & 17.50 & ns & ns & ns \\
\hline & Mean W & 17.90 & 17.50 & & & & \\
\hline \multicolumn{8}{|l|}{ Dressing percentage } \\
\hline \multirow[t]{3}{*}{ 1) $\%$ of live body wt } & FL 00 & 43.65 & 39.05 & 41.35 & 1.13 & 1.13 & 1.60 \\
\hline & FL 20 & 43.73 & 40.28 & 42.60 & $*$ & ns & ns \\
\hline & Mean W & 43.69 & 39.67 & & & & \\
\hline \multirow[t]{3}{*}{ 2) $\%$ of empty body wt } & FL 00 & 53.01 & 50.05 & 51.53 & 1.26 & 1.26 & 1.78 \\
\hline & FL 20 & 52.92 & 50.62 & 51.77 & ns & ns & ns \\
\hline & Mean W & 52.96 & 50.34 & & & & \\
\hline
\end{tabular}

FW: Fresh water; SW: Saline water; FW x FL: Fresh water + Flavomycin; SW x FL : Saline water + Flavomycin; $\quad *: \mathrm{P}<0.05 ; * *: \mathrm{P}<0.01$; ns: not significant $\mathrm{P}<0.05$

\section{Wholesale cuts :}

Wholesale cuts of carcasses expressed as a percentage of chilled carcass weight for different experimental groups are shown in Table (6).

Saline load resulted in a significant decrease in neck and tail percentages while it caused significant increase $(\mathrm{P}<0.01)$ of racks and legs percentages. Fahmy (1998) found a decrease in neck and flank percentages by salt load when fed sheep on silage of salty plants. Flavomycin increased the percentage of shoulder, but decreased leg segment. Combining SW and FL yielded large decrease in neck \%.

\section{Physical components of 9 - 10 - 11 rib cut and organoleptic test:}

Table (7) demonstrates the distribution of lean, fat and bone in the best rib cut, eye muscle area and organoleptic traits (Aroma, flavour, tenderness, juiciness and palatability) as affected by SW and FL.

Flavomycin administration had no effect on the physical composition of the best rib cut. Drinking saline water brought about significant $(\mathrm{P}<0.01)$ increase in lean meat percentage on the expense of fat percentage. Eye muscle area was not affected by either salinity of water or flavomycin addition (Table 7). 
Table 5.Least square means $\pm \mathrm{SE}$ of percentages of organs and ofals from empty body weight for Barki lambs in experimental groups

\begin{tabular}{|c|c|c|c|c|c|c|c|}
\hline \multirow[t]{2}{*}{ Item } & \multirow{2}{*}{$\begin{array}{c}\text { FL } \\
\text { Level }\end{array}$} & \multirow{2}{*}{ TW } & \multirow[b]{2}{*}{ SW } & \multirow{2}{*}{$\begin{array}{c}\text { Mean } \\
\text { FL }\end{array}$} & \multicolumn{3}{|c|}{$\pm \mathrm{SE}$} \\
\hline & & & & & $\mathbf{W}$ & FL & $\mathbf{W} \square \mathbf{F L}$ \\
\hline \multirow[t]{3}{*}{ Head } & FL 00 & 8.39 & 8.21 & 8.30 & 0.26 & 0.26 & 0.37 \\
\hline & FL 20 & 8.98 & 8.82 & 8.90 & $\mathrm{~ns}$ & ns & $\mathrm{ns}$ \\
\hline & Mean W & 8.68 & 8.51 & & & & \\
\hline \multirow[t]{3}{*}{ Four feet } & FL 00 & 2.91 & 3.16 & 3.04 & 0.14 & 0.14 & 0.20 \\
\hline & FL 20 & 2.89 & 3.09 & 2.99 & $\mathrm{~ns}$ & ns & ns \\
\hline & Mean W & 2.90 & 3.12 & & & & \\
\hline \multirow[t]{3}{*}{ Pelt } & FL 00 & 17.26 & 18.42 & 17.84 & 0.64 & 0.64 & 0.91 \\
\hline & FL 20 & 18.18 & 19.96 & 19.07 & ns & $*$ & ns \\
\hline & Mean W & 17.70 & 19.19 & & & & \\
\hline \multirow[t]{3}{*}{ Liver } & FL 00 & 1.27 & 1.34 & 1.31 & 0.08 & 0.08 & 0.11 \\
\hline & FL 20 & 1.36 & 1.36 & 1.36 & ns & ns & ns \\
\hline & Mean W & 1.32 & 1.35 & & & & \\
\hline \multirow[t]{3}{*}{ Heart } & FL 00 & 0.52 & 0.44 & 0.48 & 0.04 & 0.04 & 0.06 \\
\hline & FL 20 & 0.47 & 0.39 & 0.43 & ns & ns & ns \\
\hline & Mean W & 0.49 & 0.42 & & & & \\
\hline \multirow{3}{*}{$\begin{array}{l}\text { Lungs and } \\
\text { trachea }\end{array}$} & FL 00 & 1.53 & 1.77 & 1.65 & 0.12 & 0.12 & 0.16 \\
\hline & FL 20 & 1.73 & 1.52 & 1.63 & ns & ns & ns \\
\hline & Mean W & 1.63 & 1.65 & & & & \\
\hline \multirow[t]{3}{*}{ Kidneys } & FL 00 & 0.35 & 0.39 & 0.37 & 0.01 & 0.01 & 0.02 \\
\hline & FL 20 & 0.34 & 0.37 & 0.36 & ns & ns & ns \\
\hline & Mean W & 0.35 & 0.38 & & & & \\
\hline \multirow[t]{3}{*}{ Testicles } & FL 00 & 0.87 & 0.79 & 0.83 & 0.07 & 0.07 & 0.10 \\
\hline & FL 20 & 0.72 & 0.74 & 0.73 & ns & ns & ns \\
\hline & Mean W & 0.80 & 0.77 & & & & \\
\hline \multirow[t]{3}{*}{ Spleen } & FL 00 & 0.19 & 0.16 & 0.17 & 0.006 & 0.006 & 0.01 \\
\hline & FL 20 & 0.18 & 0.19 & 0.19 & $*$ & $* *$ & $* *$ \\
\hline & Mean W & 0.18 & 0.18 & & & & \\
\hline \multirow[t]{3}{*}{ Kidneys fat } & FL 00 & 0.32 & 0.27 & 0.29 & 0.05 & 0.05 & 0.07 \\
\hline & FL 20 & 0.39 & 0.71 & 0.40 & $\mathrm{~ns}$ & $\mathrm{~ns}$ & ns \\
\hline & Mean W & 0.35 & 0.34 & & & & \\
\hline \multirow[t]{3}{*}{ Abdominal fat } & FL 00 & 0.65 & 0.74 & 0.70 & 0.10 & 0.10 & 0.14 \\
\hline & FL 20 & 0.66 & 0.51 & 0.59 & ns & ns & ns \\
\hline & Mean W & 0.66 & 0.63 & & & & \\
\hline \multirow[t]{3}{*}{ Dig- tract full } & FL 00 & 28.65 & 37.66 & 33.17 & 2.27 & 2.27 & 3.21 \\
\hline & FL 20 & 28.22 & 33.94 & 31.08 & $* *$ & ns & ns \\
\hline & Mean W & 28.45 & 35.80 & & & & \\
\hline \multirow{3}{*}{$\begin{array}{l}\text { Dig- } \\
\text { empty }\end{array}$} & FL 00 & 7.06 & 9.03 & 8.04 & 0.33 & 0.33 & 0.47 \\
\hline & FL 20 & 7.17 & 8.45 & 7.81 & $* *$ & $\mathrm{~ns}$ & ns \\
\hline & Mean W & 7.12 & 8.74 & & & & \\
\hline
\end{tabular}

FW: Fresh water; SW: Saline water; FW x FL: Fresh water + Flavomycin; SW x FL : Saline water + Flavomycin; $\quad$ *: $\mathrm{P}<0.05 ; * *: \mathrm{P}<0.01$; ns: not significant $\mathrm{P}<$ 0.05 
Table 6. Least square means $\pm \mathrm{SE}$ of percentages of wholesale cuts (cut weight/chilled carcass weight $\mathbf{x 1 0 0 )}$ of Barki lambs for experimental groups

\begin{tabular}{|c|c|c|c|c|c|c|c|}
\hline \multirow[t]{2}{*}{ Item } & \multirow{2}{*}{$\begin{array}{c}\text { FL } \\
\text { Level }\end{array}$} & & & \multirow{2}{*}{$\begin{array}{c}\text { Mean } \\
\text { W }\end{array}$} & \multicolumn{3}{|c|}{$\pm \mathrm{SE}$} \\
\hline & & TW & SW & & W & FL & $\mathbf{W} \square \mathbf{F L}$ \\
\hline \multirow{3}{*}{$\begin{array}{l}\text { Chilled carcass wt } \\
(\mathrm{kg})\end{array}$} & FL 00 & 19.37 & 16.43 & 17.90 & 1.48 & 1.48 & 2.09 \\
\hline & FL 20 & 18.17 & 16.83 & 17.50 & Ns & $\mathrm{ns}$ & ns \\
\hline & Mean W & 18.77 & 16.63 & & & & \\
\hline \multirow[t]{3}{*}{ Neck } & FL 00 & 8.41 & 7.22 & 7.82 & 0.15 & 0.15 & 0.21 \\
\hline & FL 20 & 8.08 & 6.10 & 7.09 & $* *$ & ** & ns \\
\hline & Mean W & 8.29 & 6.66 & & & & \\
\hline \multirow{3}{*}{ Shoulders } & FL 00 & 18.37 & 18.78 & 18.57 & 0.32 & 0.32 & 0.44 \\
\hline & FL 20 & 19.87 & 19.65 & 19.76 & ns & $* *$ & ns \\
\hline & Mean W & 19.12 & 19.22 & & & & \\
\hline \multirow[t]{3}{*}{ Racks } & FL 00 & 25.23 & 27.02 & 26.13 & 0.35 & 0.35 & 0.49 \\
\hline & FL 20 & 25.26 & 26.63 & 25.94 & ** & ns & ns \\
\hline & Mean W & 25.24 & 26.83 & & & & \\
\hline \multirow{3}{*}{ Loin } & FL 00 & 6.32 & 5.65 & 5.98 & 0.33 & 0.33 & 0.47 \\
\hline & FL 20 & 6.21 & 6.37 & 6.29 & ns & ns & ns \\
\hline & Mean W & 6.26 & 6.01 & & & & \\
\hline \multirow{3}{*}{ Flanks } & FL 00 & 4.71 & 5.14 & 4.93 & 0.46 & 0.46 & 0.65 \\
\hline & FL 20 & 5.46 & 5.99 & 5.73 & ns & ns & ns \\
\hline & Mean W & 5.09 & 5.57 & & & & \\
\hline \multirow[t]{3}{*}{ Legs } & FL 00 & 31.66 & 32.98 & 32.32 & 0.60 & 0.60 & 0.85 \\
\hline & FL 20 & 31.02 & 32.79 & 31.91 & $* *$ & $\mathrm{~ns}$ & ns \\
\hline & Mean W & 31.34 & 32.89 & & & & \\
\hline \multirow[t]{3}{*}{ Tail } & FL 00 & 5.09 & 3.22 & 4.16 & 0.64 & 0.64 & 0.91 \\
\hline & FL 20 & 4.11 & 3.34 & 3.73 & $*$ & ns & ns \\
\hline & Mean W & 4.60 & 3.28 & & & & \\
\hline
\end{tabular}

FW: Fresh water; SW: Saline water; FW x FL: Fresh water + Flavomycin; SW x FL:

Saline water+ Flavomycin; $\quad *$ : $\mathrm{P}<0.05 ; * *: \mathrm{P}<0.01$; ns: not significant $\mathrm{P}<0.05$

Mohamed (1997) found in rabbits an increase in carcass muscle \% and a decrease in fat and bone percentage by drinking Sw of tolerable concentration (3369 ppm TDS). In sheep, Fahmy (1998) reported an increase in the longissimus dorsi muscle area between $9^{\text {th }}$ and $10^{\text {th }}$ rib from $6.96 \mathrm{~cm}^{2}$ to $20.7 \mathrm{~cm}^{2}$ by feeding the silage of halophytic shrubs. However, he found that the lean percentage significantly decreased in leg and rack cuts, while the fat percentage significantly incresed in all prime cuts. Saline water improved, in general, the traits of meat acceptability as indicated by the significantly $(\mathrm{P}<0.01)$ higher scores assigned to the samples from aninals drank saline water than those from those drank fresh water. flavomycin addition with saline water scored higher grades than those flavomycin with fresh water.

In conclusion, adminstration of flavomycin to growing lambs raised under desert conditions could reduce some negative effects of salt stress on growth performance and carcass traits. 
Table 7. Least square means $\pm \mathrm{SE}$ of percentages of physical components of 910-11 ribs and oraganoleptic traits of Barki lambs for experimental groups

\begin{tabular}{|c|c|c|c|c|c|c|c|}
\hline \multirow[t]{2}{*}{ Item } & \multirow{2}{*}{$\begin{array}{c}\text { FL } \\
\text { Level }\end{array}$} & & & \multirow{2}{*}{$\begin{array}{l}\text { Mean } \\
\text { FL }\end{array}$} & \multicolumn{3}{|c|}{$\pm \mathbf{S E}$} \\
\hline & & TW & SW & & $\mathbf{W}$ & FL & $\mathbf{W} \square \mathbf{F L}$ \\
\hline \multirow{3}{*}{$\begin{array}{l}\text { 9-10-11 rib cut wt } \\
\text { (g) }\end{array}$} & FL 00 & 0.890 & 0.750 & 0.820 & 0.06 & 0.06 & 0.09 \\
\hline & FL 20 & 0.770 & 0.803 & 0.787 & $\mathrm{~ns}$ & ns & ns \\
\hline & Mean W & 0.830 & 0.777 & & & & \\
\hline \multirow[t]{3}{*}{ Lean meat $\%$} & FL 00 & 52.55 & 63.35 & 57.95 & 1.29 & 1.29 & 1.83 \\
\hline & FL 20 & 52.92 & 62.09 & 57.51 & $* *$ & ns & $\mathrm{ns}$ \\
\hline & Mean W & 52.73 & 62.72 & & & & \\
\hline \multirow[t]{3}{*}{ Bone \% } & FL 00 & 25.97 & 26.95 & 26.48 & 1.15 & 1.15 & 1.63 \\
\hline & FL 20 & 26.74 & 25.08 & 25.91 & ns & ns & $\mathrm{ns}$ \\
\hline & Mean W & 26.36 & 26.03 & & & & \\
\hline \multirow[t]{3}{*}{ Fat $\%$} & FL 00 & 21.48 & 9.66 & 15.57 & 1.48 & 1.48 & 2.09 \\
\hline & FL 20 & 20.14 & 12.56 & 16.35 & $* *$ & ns & $\mathrm{ns}$ \\
\hline & Mean W & 20.81 & 11.11 & & & & \\
\hline \multirow[t]{3}{*}{ Lean: Bone ratio } & FL 00 & 2.05 & 2.37 & 2.21 & 0.12 & 0.12 & 0.16 \\
\hline & FL 20 & 1.99 & 2.33 & 2.16 & $* *$ & ns & $\mathrm{ns}$ \\
\hline & Mean W & 2.02 & 2.35 & & & & \\
\hline \multirow[t]{3}{*}{ Lean: Fat ratio } & FL 00 & 2.57 & 6.82 & 4.70 & 0.42 & 0.42 & 0.60 \\
\hline & FL 20 & 2.76 & 4.87 & 3.82 & $*$ & ns & ns \\
\hline & Mean W & 2.67 & 5.85 & & & & \\
\hline \multirow[t]{3}{*}{$\mathrm{CM}$} & FL 00 & 2.93 & 2.74 & 2.83 & 0.17 & 0.17 & 0.23 \\
\hline & FL 20 & 2.76 & 3.00 & 2.88 & ns & ns & $\mathrm{ns}$ \\
\hline & Mean W & 2.84 & 2.87 & & & & \\
\hline \multirow{3}{*}{$\begin{array}{l}\text { Eye muscle area } \\
\left(\mathrm{cm}^{2}\right)\end{array}$} & FL 00 & 15.47 & 12.83 & 14.15 & 1.43 & 1.43 & 2.02 \\
\hline & FL 20 & 15.30 & 12.93 & 14.12 & ns & Ns & ns \\
\hline & Mean W & 15.38 & 12.88 & & & & \\
\hline \multirow{3}{*}{ Cooking loss } & FL 00 & 32.75 & 34.03 & 33.39 & 0.91 & 0.91 & 1.28 \\
\hline & FL 20 & 34.62 & 35.03 & 34.82 & $* *$ & $*$ & $*$ \\
\hline & Mean W & 33.69 & 34.53 & & & & \\
\hline \multicolumn{8}{|c|}{ Oraganoleptic traits of meat } \\
\hline \multirow[t]{3}{*}{ Aroma } & FL 00 & 3.90 & 4.27 & 4.08 & 0.06 & 0.06 & 0.09 \\
\hline & FL 20 & 3.68 & 4.00 & 3.84 & $* *$ & $* *$ & Ns \\
\hline & Mean W & 3.79 & 4.12 & & & & \\
\hline \multirow[t]{3}{*}{ Flavour } & FL 00 & 3.85 & 4.00 & 3.92 & 0.06 & 0.06 & 0.09 \\
\hline & FL 20 & 3.62 & 4.12 & 3.87 & $* *$ & ns & $*$ \\
\hline & Mean W & 3.73 & 4.06 & & & & \\
\hline \multirow[t]{3}{*}{ Tenderness } & FL 00 & 3.83 & 4.07 & 3.95 & 0.07 & 0.07 & 0.09 \\
\hline & FL 20 & 3.40 & 3.90 & 3.65 & $* *$ & $* *$ & $\mathrm{~ns}$ \\
\hline & Mean W & 3.62 & 3.98 & & & & \\
\hline \multirow[t]{3}{*}{ Juicciness } & FL 00 & 3.68 & 3.88 & 3.78 & 0.06 & 0.06 & 0.08 \\
\hline & FL 20 & 3.52 & 3.83 & 3.68 & $* *$ & ns & ns \\
\hline & Mean W & 3.60 & 3.86 & & & & \\
\hline \multirow[t]{3}{*}{ Palatability } & FL 00 & 3.88 & 4.03 & 3.96 & 0.04 & 0.04 & 0.060 \\
\hline & FL 20 & 3.60 & 4.02 & 3.81 & $* *$ & $*$ & $*$ \\
\hline & Mean W & 3.74 & 4.03 & & & & \\
\hline
\end{tabular}

FW: Fresh water; SW: Saline water; FW x FL: Fresh water + Flavomycin; SW x FL : Saline water + Flavomycin; $\quad *$ : $\mathrm{P}<0.05 ; * *: \mathrm{P}<0.01 ;$ ns: not significant $\mathrm{P}<0.05$ 


\section{REFERENCES}

Abd-El-Aziz, M.M., 1983. A study on the biological efficiency of meat production in Barki sheep. M.Sc. Thesis, Fac. Agric., Moshtohor, Zagazig Univ., Benha Branch.

Aboul-Naga, A.M.and M.B. Aboul- Ela, 1986. Performance of subtropical Egyptian sheep breeds, Europian breeds and their crosses. 1- Egyptian sheep breeds. World Review of Animal Production; XX111 (1): 75.

Aggour, T.A., 1990. Responses of geomorphologic and geological feature on ground water in Wadi Araba, Eastern Desert, Egypt. M.Sc. Thesis, Fac.Sci., Ain Shams Univ.

Ahmed, M.H., 1996.Effect of saline water on productive performance of rabbits. M.Sc. Thesis, Faculty of Agric., Zagazig Univ.

A.O.A.C., 1990. Official Methods of Analysis of the Association of Official Agricultural Chemists. Wash.ington, D.C., USA.

Ayyat, M.S., A.A. Habeeb and S.M. Bassuny, 1991. Effect of water salinity on growth performance, carcass traits and some physiological aspects of growing rabbits in summer season. Egyptian J. of Rabbit Science, 1:21.

Davey, L.A. 1980.The national use of feed additives in animal production. 31, Hahrestagung der Europaischen Vereiningung fur Tierzucht, 1-4 September, Munchen, Germany.PP: 1-9.

El-Basiony, A.Z., 1994. Performance of growing lambs and buffalo calves given flavomycin as a feed supplement. Annals of Agric. Sci., Moshtohor, 32:1791.

El-Sherif, M.M.A., 1991. Growth performance of lambs as affected by their thermoregulation characteristics. Ph. D. Thesis. Faculty of Agric. Cario. Univ.

El-Sherif, M.M.A and E.E. El-Hassanein, 1996. Influence of drinking saline water on growth and distribution of body fluids in sheep. Alex. J. Agric. Res. 41(3):1-9.

El-Sherif, M.M.A, E.E. Tharwat, S.O. Amin, M.M.E. Ahamed and A.H. Hammam, 2001. Effect of high level of feeding and growth promoters on thermal balance and growth performance of Barki ewe lambs under northwestern desert conditions. Journal of Environmental Science; 2(2): 323-352.

Fahmy, A.A., 1998. Nutritional studies on halophytes and agricultural wastes as feed supplements for small ruminants in Sinai. Ph.D. Thesis, Fac. Agric. Cario Univ.

Hamada, M.K.O., 1976. Mutton and wool production. Dar El-Maaref, Alexandria, Egypt (in Arabic).

Hammam, A.H., 2001. Improving growth and productivity of ewe lambs under the enviromental conditions of North Western Desert.Ph.D. Thesis, Fac. Agric. Cario Univ.

Ismail, S.A.A., 2003. Nutritional evaluation of the effect of flavomycin and saline water on nutrient digestibility, lamb performance and some carcass traits. Egyptian J. of Nutrition and Feeds .

Kearl, L.C., 1982. Nutrient Requirements of Ruminants in Developing Countries. International Feedstuffs Institute. Utah Agric. Exp. Stn., Utah State.

Mohamed, A.M.H., 1997. Study of some physiological, nutritional and productive aspects of rabbits as affected by natural saline water. M. Sc. Thesis, Fac. Agric. Menoufia Univ. 
Morris, F.E., M.E. Branine, M.L. Galyeean, M.E. Hubbert, A.S. Freema and G.P. Lafgree, 1990. Effect of rotating monensin plus tylosin and lasalocid in performance, ruminal fermentation and sit and extent of digestion in feedlot cattle. J.Anim. Sci., 63:3069.

Murray, P.J., S.G. Winslow and J.B. Rowe, 1992. Conditions under which flavomycin increases wool growth and live weight gain in sheep. Australian J. Agric. Res., 43: (2)367.

Paitial, N.V., G.B. Deshumkh, J.D. Hanmode, D.H. Rekhate and R.C. Takarkhede, 1996. Effect of monensin on growth rate of Malpure lambs. Livestock Adviser; 21(5): 3-10.

Pierce, A.W., 1966. Studies on salt tolerance of sheep. V1: The tolerance of weathers in pens for drinking water of the types obtained from underground water sources in Australia. Aust. J. Agric. Res. 17:209-218.

S.A.S., 1985. SAS User's Guide: Statistics (version 5 ed.). SAS Inst. Inc., Cary, N.C.

Shawkat, Safinaz, M.; H.M. Abou El Nasr; M.F.A. Farid and H.M. El-Shaer (1988). Studies on saline drinking water for camels and sheep. II. Feed utilization and nitrogen balance. Alex. J. Agric. Res., 33:107

Singh, N.P. and G.C. Taneja, 1979. Effect of prolonged salt intake from drinking water on the distribution of body water in Mawari sheep of the Rajasthan Desert. Anim. Prod., 27:357

Spires, H.R., E. Olmsted, L.L. Berger, J.P. Sontenot, D.R. Gill, J.G. Riley, M.I. Wray and R.A. Zinn, 1990. Efficiency of Laidlomycin propionate for increasing rate and efficiency of gain by cattle. J. Aninal Sci,; 68: 3382-3391. 
تأثير شرب الماء المالح وإعطاء الفلافوميسين على أداء النمو وصفات الذبيحة للأغنام البرقى

محمد فرج شحاتة و أحمد حسين همام مركز بحوث الصحراء - الدطرية - الثقاهة

أستخدم فى هذه الدراسة أربعون حولى برقى نامى (عمر ستة أثهر منوسط وزن ..4 كجم) قسمت

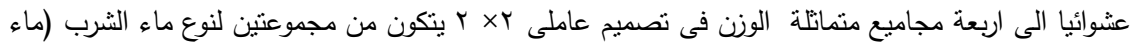

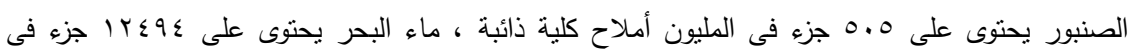

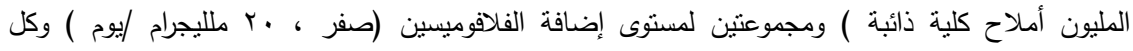

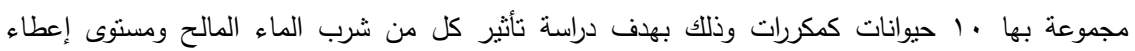

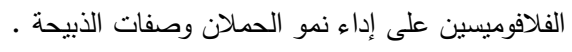

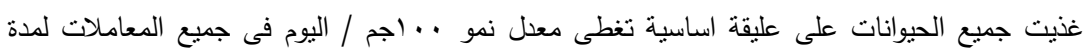

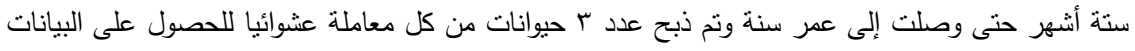
الخاصة بمواصفات الذبيحة.

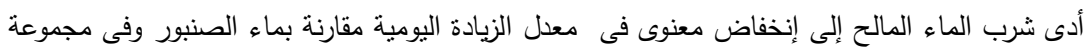

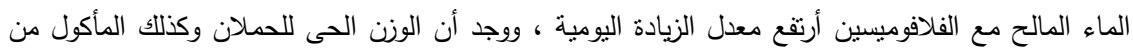

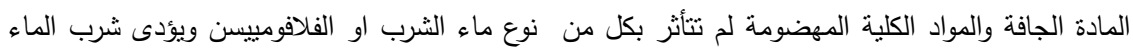

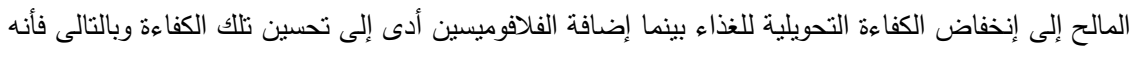

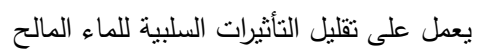

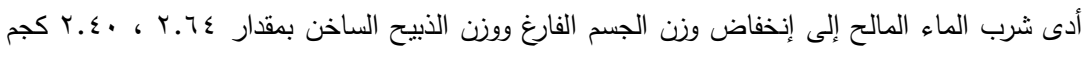

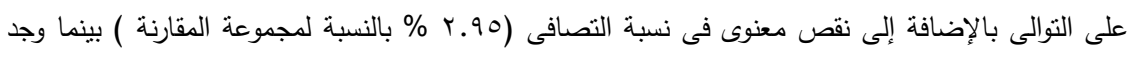

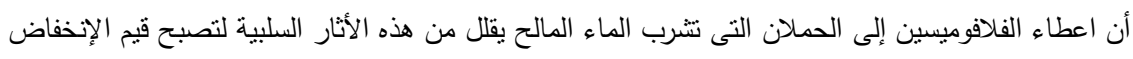

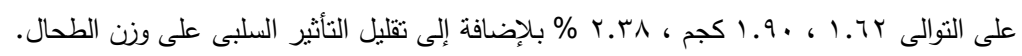

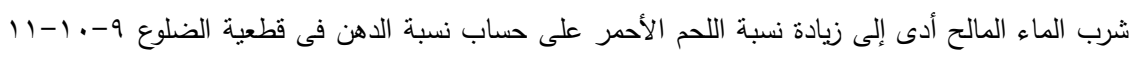

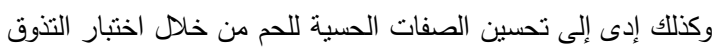

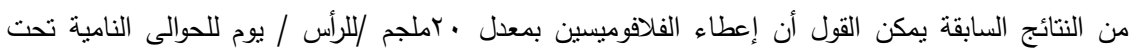

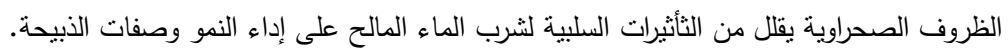

